\title{
Ruptured Interstitial Ectopic Pregnancy at 18 Weeks: How Failure of Early Detection Resulted in a Maternal Near-miss
}

\author{
David Putiero Suoseg ${ }^{1}$ *, Anwar Sadat Seidu ${ }^{3}$, Ubeida Koray ${ }^{1}$, Safia Iddrisu ${ }^{1}$, \\ Munkaila Mohammed ${ }^{1}$, Shafawu Mustapha Baba ${ }^{1}$, Abraham Babatuiamu Titigah ${ }^{1}$, \\ Ana Maria Simono Charadan ${ }^{1,2}$ \\ ${ }^{1}$ Department of Obstetrics and Gynecology, Tamale Teaching Hospital, Tamale, Ghana \\ ${ }^{2}$ Department of Obstetrics and Gynecology, School of Medicine and Health Sciences, University for Development Studies, Tamale, Ghana \\ ${ }^{3}$ Department of Surgery, Tamale Teaching Hospital, Tamale, Ghana
}

\section{Email address:}

dsuoseg@gmail.com (D. P. Suoseg)

${ }^{*}$ Corresponding author

\section{To cite this article:}

David Putiero Suoseg, Anwar Sadat Seidu, Ubeida Koray, Safia Iddrisu, Munkaila Mohammed, Shafawu Mustapha Baba, Abraham Babatuiamu Titigah, Ana Maria Simono Charadan. Ruptured Interstitial Ectopic Pregnancy at 18 Weeks: How Failure of Early Detection Resulted in a Maternal Near-miss. Journal of Gynecology and Obstetrics. Vol. 9, No. 4, 2021, pp. 125-127. doi: 10.11648/j.jgo.20210904.16

Received: August 6, 2021; Accepted: August 19, 2021; Published: August 27, 2021

\begin{abstract}
Interstitial ectopic pregnancy occurs when a gestational sac implants within the interstitial portion of the Fallopian tube. It is an uncommon and dangerous type of ectopic pregnancy with high morbidity and mortality. Rupture of interstitial ectopic pregnancy usually leads to life-threatening hemorrhage. This case report illustrates how failure of early detection of interstitial ectopic pregnancy resulted in a maternal near-miss and calls for the need for increased awareness and high index of suspicion among sonographers and clinicians. A case of a 24-year-old gravida two para zero, one previous ectopic pregnancy $\left(\mathrm{G} 2 \mathrm{P} 0^{1 \mathrm{EP}}\right)$ was brought into our facility with generalized abdominal pain and hemorrhagic shock. She had a previous history of left salpingectomy for a ruptured tubal ectopic pregnancy four years earlier at a secondary health care facility. Emergency laparotomy after aggressive resuscitation revealed ruptured interstitial ectopic pregnancy with massive hemoperitoneum and a dead fetus. Wedge resection of the right cornua along with the Fallopian tube was done. Diagnosis of interstitial ectopic pregnancy is challenging due to its rarity and location in the intrauterine portion of the Fallopian tube. Ultrasonographic diagnosis in early pregnancy is key. Awareness creation and a high index of suspicion is required among sonographers and clinicians to prevent needless morbidity and mortality.
\end{abstract}

Keywords: Ectopic, Hemorrhagic Shock, Interstitial, Life-threatening, Near-miss

\section{Introduction}

Interstitial ectopic pregnancy is defined as the implantation of a gestational sac within the interstitial part of the Fallopian tube. The interstitial part is the most proximal portion of the Fallopian tube that lies within the myometrium. This portion of the tube is about $2 \mathrm{~cm}$ long and $0.7 \mathrm{~mm}$ wide and is described as intrauterine but lies outside the endometrial cavity $[1,2]$.

Ruptured interstitial ectopic pregnancy has the highest risk of life-threatening hemorrhage because of the proximity of the site of implantation to the uterine and ovarian vessels [3].

Predisposing factors for interstitial ectopic pregnancy are like other ectopic pregnancies. These include; previous tubal pregnancies, pelvic surgeries, previous intrauterine procedures, in vitro fertilization, previous pelvic inflammatory diseases and sexually transmitted diseases [4].

In our setting, it is unusual to make a diagnosis of unruptured ectopic pregnancy in general due to factors such as late presentation of pregnant women to health facilities and failure of clinicians to recognize clinical features of ectopic pregnancy [5]. Interstitial ectopic pregnancy is even more difficult to diagnose as both presenting features and available imaging techniques are less sensitive and specific [6].

This case report is intended to create awareness and raise 
the index of suspicion among clinicians of interstitial ectopic pregnancy and its diagnostic challenges.

\section{Case Presentation}

A 24-year-old $\mathrm{G} 2 \mathrm{P} 0^{1 \mathrm{EP}}$ was brought to our facility from a peripheral health care facility as a case of severe anemia in pregnancy and hypovolemic shock. About 2 hours prior to presentation, she started experiencing sudden onset of lower abdominal pain which progressively worsened and associated with dizziness. She was sent to the peripheral health care facility where resuscitation was started and referred to our facility.

Client had a laparotomy and left salpingectomy for a ruptured left ectopic gestation 4 years earlier in a primary health facility. There was no significant family history. Her last menstrual period was 5 months ago. She had a positive home pregnancy test two months after she missed her menses. An obstetric ultrasonography (USG) done two weeks prior to presentation indicated an intrauterine pregnancy at 16 weeks +2 days with active fetal cardiac activity.

On arrival at our facility, the patient was in hypovolemic shock, lethargic, very pale with cold clammy extremities. The pulse was barely palpable, blood pressure was $40 / 25 \mathrm{mmHg}$. The abdomen was distended with generalized tenderness. On pelvic examination, the vulvovaginum was clean. There was mild cervical excitation tenderness on the right. the cervix was closed and appeared normal.

Bedside USG showed massive hemoperitoneum, an 18 weeks +5 days fetus with no fetal cardiac activity in the peritoneum, adnexa was clear and an empty bulky uterus. A diagnosis of massive hemoperitoneum due to ruptured ectopic gestation with differential of a ruptured uterus was made.

She was resuscitated with crystalloids and optimized for emergency exploratory laparotomy. Baseline laboratory investigations were done and grouping and cross-matching against 4-6 units of blood.

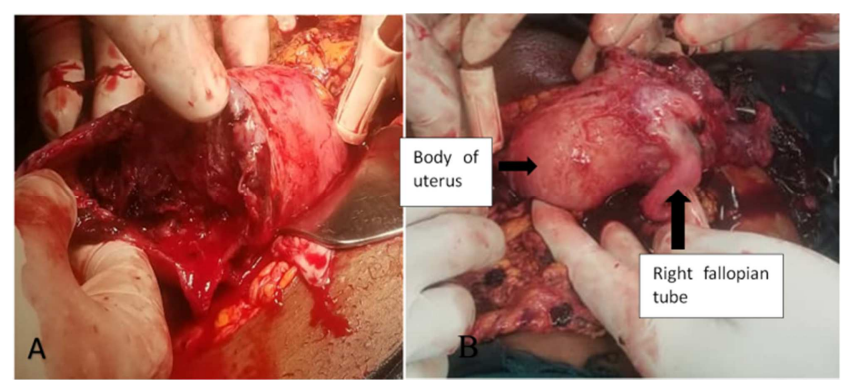

Figure 1. Jpg; A) showing the body of uterus and ruptured anterior surface of right fundus B) showing posterior surface of body of uterus and right Fallopian tube.

Intra-operatively: Massive hemoperitoneum of about 3L, fetus expelled from the ruptured right cornua with the placenta still attached to the cavity. There was no visible opening connecting the ruptured portion to the endometrial cavity. There was an ipsilateral right fallopian tube attached to the ruptured portion with a medially located right round ligament. The entire left fallopian tube was absent indicating previous left total salpingectomy. Both ovaries were present and of normal size. Resection of the right cornua along with the right fallopian tube was done. Hemostasis was secured. She received 3 units of whole blood intra-operatively.

Surgery was uneventful, immediate post-operative condition was satisfactory. She received 2 extra units of whole blood and was discharged after 3 days.

She was counselled on available fertility options.

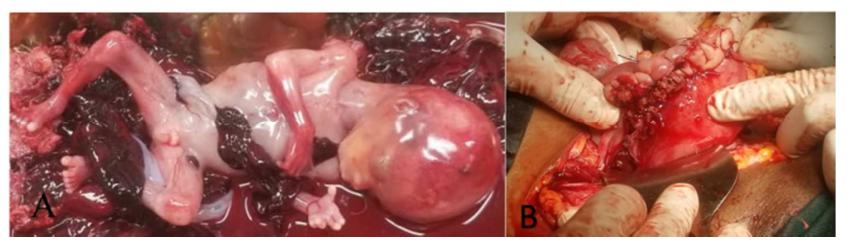

Figure 2. Jpg; A) fetus with placenta B) uterine repair after wedge-resection.

\section{Discussion}

Interstitial ectopic pregnancy is an uncommon and most fatal type of ectopic pregnancy. It accounts for $2-4 \%$ of all ectopic gestation [4], with up to $2-2.5 \%$ mortality [7]. It was believed that most interstitial ectopic pregnancies rupture late in the pregnancy because of the ability of the surrounding myometrium to distend and accommodate the growing gestation until the second trimester [8]. That belief has been challenged by recent case series which reported rupture of interstitial ectopic pregnancy earlier than 12 weeks of gestation [9]. Notwithstanding, there are known cases of interstitial ectopic pregnancy which progressed near term or to term [10].

Interstitial ectopic pregnancy may be misdiagnosed as angular or cornual pregnancy and that can lead to fatal consequences as the clinical progression and management differ. Angular pregnancy is defined as an implanted embryo at one of the lateral angles of the endometrial cavity, medial to the utero-tubal junction, and the round ligament. It is not an ectopic pregnancy and does not pose any danger to the patient. Cornual pregnancy on the other hand, is the implantation of an embryo in one of the horns of a bicornuate uterus, a septate uterus or in a rudimentary horn of a unicornuate uterus. The first two implantations are not ectopic pregnancies while the latter is a form of an ectopic pregnancy [2].

Ruptured interstitial ectopic pregnancy usually results in acute torrential bleeding which can be life-threatening [4]. The acute torrential bleeding most likely accounted for the rapid deterioration of our patient within two hours from the onset of abdominal pain to presentation at our facility. Clinicians need to have a high index of suspicion for early diagnosis and management to decrease morbidity and mortality. Most women with small unruptured ectopic gestation have no significant clinical findings [1]. The classic triad of amenorrhea followed by vaginal bleeding and abdominal pain on the affected side that occurs in ectopic pregnancy are only present in less than $40 \%$ of patients [6].

Pre-rupture investigative modalities include; serum betahuman chorionic gonadotropin (beta-hCG), ultrasound, 
laparoscopy, hysteroscopy, and magnetic resonance imaging (MRI). Most of these investigative modalities are unavailable in many areas of low-resource settings like ours. Therefore, in the presence of identifiable risk factors such as previous salpingectomy as in our case, the use of ultrasound (preferably transvaginal) and serum beta-hCG estimation which are readily available in most settings are valuable for the early diagnosis of interstitial ectopic pregnancy. However, the sonographic diagnosis of interstitial pregnancy can be challenging because of its location in the intrauterine segment of the Fallopian tube, and its invasion of the surrounding myometrium. This makes it difficult to distinguish it from a normal intrauterine pregnancy especially in an inexperienced hand [6]. It is even more difficult in the second trimester as the increasing size of the gestational sac makes the ultrasonographic diagnosis more obscure [11]. In this case, the patient had an ultrasound done at 16 weeks gestation which reported a normal intrauterine gestation.

Timor-Tritsch et al proposed the following criteria for the ultrasonographic diagnosis of interstitial ectopic pregnancy: an empty uterine cavity, a separate gestational sac more than $1 \mathrm{~cm}$ from the most lateral edge of the uterine cavity and a thin myometrial layer $(\leq 5 \mathrm{~mm})$ surrounding the gestational sac [12].

Ackerman et al, describes the presence of an echogenic line that extends from the endometrial cavity to and abutting the interstitial mass or gestational sac, referred to as the interstitial line sign. It has a high sensitivity and specificity for interstitial ectopic pregnancy [13].

Conservative techniques are increasingly being used in the management of interstitial ectopic pregnancy with hemodynamic stability because of early diagnosis [4]. Conservative approaches include; hysteroscopic removal of gestational sac, laparoscopic cornuostomy, cornual resection or salpingotomy [4]. Laparotomy with cornuectomy or hysterectomy is indicated when patients present with hemodynamic instability [14]. Medically, the administration of methotrexate locally, systemic or both, is effective with an overall success rate of $83 \%$ [4]. In our settings where unruptured ectopic pregnancy is rarely diagnosed, most women with interstitial ectopic pregnancy would present after rupture with hemorrhage which would require laparotomy as happened in our case.

\section{Conclusion}

Interstitial ectopic pregnancy poses diagnostic challenges due to its rarity and intrauterine location. The use of ultrasound early in pregnancy especially in low resource settings is key to early detection of interstitial ectopic pregnancy. This emphasizes the need for increased awareness and a high index of suspicion among sonographers and clinicians in order to avert needless morbidity and mortality.

\section{Conflict of Interest}

The authors declare that they have no competing interests.

\section{References}

[1] Cunningham FG, Leveno KJ, Bloom SL, Spong CY, Dashe JS, Hoffman BL, et al. Ectopic Pregnancy. In: Williams Obstetrics. 24th ed. New York, NY: McGraw-Hill Education; 2014. P. $387-8$.

[2] Finlinson AR, Bollig KJ, Schust DJ. Differentiating pregnancies near the uterotubal junction (angular, cornual, and interstitial): a review and recommendations. Fertil Res Pract. 2020 May 4; 6 (1): 8.

[3] Brincat M, Bryant-Smith A, Holland TK. The diagnosis and management of interstitial ectopic pregnancies: a review. Gynecol Surg. 2019 Feb 4; 16 (1): 2.

[4] Lau S, Tulandi T. Conservative medical and surgical management of interstitial ectopic pregnancy. Fertil Steril. 1999 Aug; 72 (2): 207-15.

[5] Obed S. Diagnosis of Unruptured Ectopic Pregnancy is Still Uncommon in Ghana. Ghana Med J. 2006 Mar; 40 (1): 3-7.

[6] Dagar M, Srivastava M, Ganguli I, Bhardwaj P, Sharma N, Chawla D. Interstitial and Cornual Ectopic Pregnancy: Conservative Surgical and Medical Management. J Obstet Gynecol India. 2018 Dec; 68 (6): 471-6.

[7] Rock JA, Jones HW. Ectopic pregnancy. In: Te Linde's Operative Gynecology. 10th ed. Philadelphia: Lippincott Williams \& Wilkins; 2008, p. 816.

[8] Kun WM, Tung WK. On the look-out for a rarity-interstitial/cornual pregnancy. Eur J Emerg Med Off J Eur Soc Emerg Med. 2001 Jun; 8 (2): 147-50.

[9] Tulandi T, Al-Jaroudi D. Interstitial pregnancy: results generated from the Society of Reproductive Surgeons Registry. Obstet Gynecol. 2004 Jan; 103 (1): 47-50.

[10] $\mathrm{Ng}$ PH, NorAzlin MI, Nasri NI. Term interstitial pregnancy with uterine conservation. Int J Gynaecol Obstet off Organ Int Fed Gynaecol Obstet. 2007 Dec; 99 (3): 251.

[11] Hamouda ES, Littooij AS, Thia EW, Ong CL. Ruptured Interstitial Ectopic Pregnancy at 18 Weeks Gestation Diagnosed by MRI: A Case Report. J Radiol Case Rep. 2013 Oct 1; 7 (10): 34-42.

[12] Timor-Tritsch IE, Monteagudo A, Matera C, Veit CR. Sonographic evolution of cornual pregnancies treated without surgery. Obstet Gynecol. 1992 Jun; 79 (6): 1044-9.

[13] Ackerman TE, Levi CS, Dashefsky SM, Holt SC, Lindsay DJ. Interstitial line: sonographic finding in interstitial [cornual] ectopic pregnancy. Radiology. 1993 Oct; 189 (1): 83-7.

[14] Rastogi R, GL M, Rastogi N, Rastogi V. Interstitial ectopic pregnancy: A rare and difficult clinicosonographic diagnosis. $\mathrm{J}$ Hum Reprod Sci. 2008; 1 (2): 81-2. 